\title{
Effectiveness of joint mobilisation after cast immobilisation for ankle fracture: a protocol for a randomised controlled trial [ACTRNOI 2605000 I 43628]
}

\author{
C Christine Lin*1, Anne M Moseley ${ }^{1}$, Kathryn M Refshauge ${ }^{1}$, Marion Haas ${ }^{2}$ \\ and Robert D Herbert ${ }^{1}$
}

Address: ${ }^{1}$ School of Physiotherapy, the University of Sydney, PO Box 170, Lidcombe, New South Wales 1825, Australia and ${ }^{2}$ Centre for Health Economics Research and Evaluation (CHERE), University of Technology, Sydney, PO Box 123, Broadway, New South Wales 2007, Australia

Email: C Christine Lin* - c.lin@usyd.edu.au; Anne M Moseley - amoseley@mail.usyd.edu.au; Kathryn M Refshauge - k.refshauge@fhs.usyd.edu.au; Marion Haas - marion.haas@chere.uts.edu.au; Robert D Herbert - r.herbert@fhs.usyd.edu.au

* Corresponding author

Published: 26 May 2006

BMC Musculoskeletal Disorders 2006, 7:46 doi:10.1 I86/147/-2474-7-46

This article is available from: http://www.biomedcentral.com/I47/-2474/7/46

(c) 2006 Lin et al; licensee BioMed Central Ltd.

This is an Open Access article distributed under the terms of the Creative Commons Attribution License (http://creativecommons.org/licenses/by/2.0), which permits unrestricted use, distribution, and reproduction in any medium, provided the original work is properly cited.
Received: 04 April 2006

Accepted: 26 May 2006

\begin{abstract}
Background: Passive joint mobilisation is a technique frequently used by physiotherapists to reduce pain, improve joint movement and facilitate a return to activities after injury, but its use after ankle fracture is currently based on limited evidence. The primary aim of this trial is to determine if adding joint mobilisation to a standard exercise programme is effective and cost-effective after cast immobilisation for ankle fracture in adults.

Methods/Design: Ninety participants will be recruited from the physiotherapy departments of three teaching hospitals and randomly allocated to treatment or control groups using a concealed procedure. All participants will perform an exercise programme. Participants in the treatment group will also receive joint mobilisation twice a week for four weeks. Blinded follow-up assessments will be conducted four, 12 and 24 weeks after randomisation. The primary outcome measures will be the Lower Extremity Functional Scale and the Assessment of Quality of Life. Secondary outcomes will include measures of impairments, activity limitation and participation. Data on the use of physiotherapy services and participants' out-of-pocket costs will be collected for the cost-effective and cost-utility analyses. To test the effects of treatment, between-group differences will be examined with analysis of covariance using a regression approach. The primary conclusions will be based on the four-week follow-up data.
\end{abstract}

Discussion: This trial incorporates features known to minimise bias. It uses a pragmatic design to reflect clinical practice and maximise generalisability. Results from this trial will contribute to an evidence-based approach for rehabilitation after ankle fracture.

\section{Background}

Ankle fracture refers to fracture of the medial or lateral malleolus or the distal tibia or fibula [1-4]. The incidence is between 107 and 184 per 100,000 person-years [5-8], making it one of the most common lower limb fractures $[9,10]$. It is usually caused by low-velocity trauma such as 
falls, twisting injuries and sports injuries [6-8], and therefore is prevalent in not only the older, but also the young and active population $[6,8]$. Orthopaedic management in adults with ankle fracture may involve reduction, surgical fixation, and immobilisation for six to 12 weeks depending on the severity of the fracture [3,4,11-13]. Not surprisingly, fracture severity has been reported to influence functional outcomes after ankle fracture $[9,14,15]$.

Physiotherapists often contribute to the rehabilitation of ankle fracture, which typically starts soon after the period of immobilisation. Due to effects of the fracture and the subsequent immobilisation, most people experience pain, swelling, stiffness, muscle atrophy and decreased muscle torque at the ankle following cast removal [1619]. Consequently, they complain of limitations in activities involving the lower limb, such as stair climbing and walking [18], and reduced participation in work and recreation [20-22].

Passive joint mobilisation is a technique commonly used by physiotherapists to address the problems of pain and joint stiffness, in order to allow an earlier return to activities. It involves the physiotherapist manually gliding the articular surfaces of a joint to produce oscillatory movements [23]. It has been proposed that manual therapy such as joint mobilisation produces analgesic effects and increases elasticity of joint structures through interactions at the local, central nervous system and psychological levels [24]. These hypotheses are yet to be validated [25]. Research to-date indicates that the analgesic effect of peripheral joint mobilisation may be short lasting [26], is not mediated by endorphins [27], and can been seen with concurrent excitation of the sympathetic nervous system $[28,29]$.

The effectiveness of joint mobilisation after post-fracture immobilisation has been investigated in a small number of trials with contrasting results. Randall et al [30] showed that in patients with metacarpophalangeal fracture, mobilisation and exercise led to a greater increase in joint movement than exercise alone. Coyle and Robertson [31] reported that mobilisation of the wrist improved pain and extension movement after Colles' fracture, but the results were not compared to a control group. Two other studies of mobilisation after Colles' fracture found no significant differences between outcomes of treatment and control groups [32,33].

There is positive evidence to support the use of joint mobilisation after acute ankle sprain [34,35]. Joint mobilisation led to increased ankle movement, improved gait patterns, reduced pain and the number of treatment sessions required, and hastened return to activities when compared with the usual rest, ice, compression and eleva- tion protocol [35]. However, the effectiveness of joint mobilisation after ankle fracture is less certain. Wilson [36] conducted a randomised pilot study to investigate if joint mobilisation plus whirlpool therapy and exercise was more effective than whirlpool therapy and exercise alone. Although an improvement in ankle movement and function was reported when the treatment group was compared to the control group, the difference between groups was not statistically significant, potentially because of the small sample size $(n=10)$.

The treatment cost of ankle fracture has been estimated in studies from Britain and Canada $[37,38]$, but to-date there are no randomised controlled trials. In a retrospective audit, costs of hospitalisation were significantly greater in those who were operated on within 24 hours, compared to those who were operated on more than 24 hours after presentation [37]. The average extra cost per patient was $£ 990$ (1998 British pounds). A prospective pilot study reported that the total hospitalisation and outpatient medical costs were $\$ 2,134$ (United States dollars) per patient over a 12-month period [38]. But this study did not include a control group, and hence no comparisons of costs or quality of life measures. Neither of these studies assessed utilisation of services or included the cost of outpatient physiotherapy. Only one published study has reported an economic analysis of joint mobilisation [39], but the comparisons made were between high-grade and low-grade mobilisation for the shoulder. Therefore, despite the common use of joint mobilisation as a treatment technique, its current use after ankle fracture is based on clinical experience and limited evidence of effectiveness and cost-effectiveness.

The primary aim of this trial is to determine whether adding joint mobilisation to an exercise programme is more effective and cost-effective than exercise alone after cast immobilisation for ankle fracture in adults. A secondary aim is to determine if treatment effects are influenced by fracture severity.

\section{Methods \\ Design}

This will be an assessor-blinded, multi-centre, randomised controlled trial (Figure 1). Participants will be stratified by site and randomly allocated in permuted blocks to treatment or control groups. The randomisation sequence will be generated a priori using the random number function in Excel by an independent investigator not directly involved in the assessment and treatment of participants. Allocations will be sealed in opaque and consecutively numbered envelopes, which will be opened in sequence by the treating physiotherapist after the recruitment and baseline assessment of each participant. 


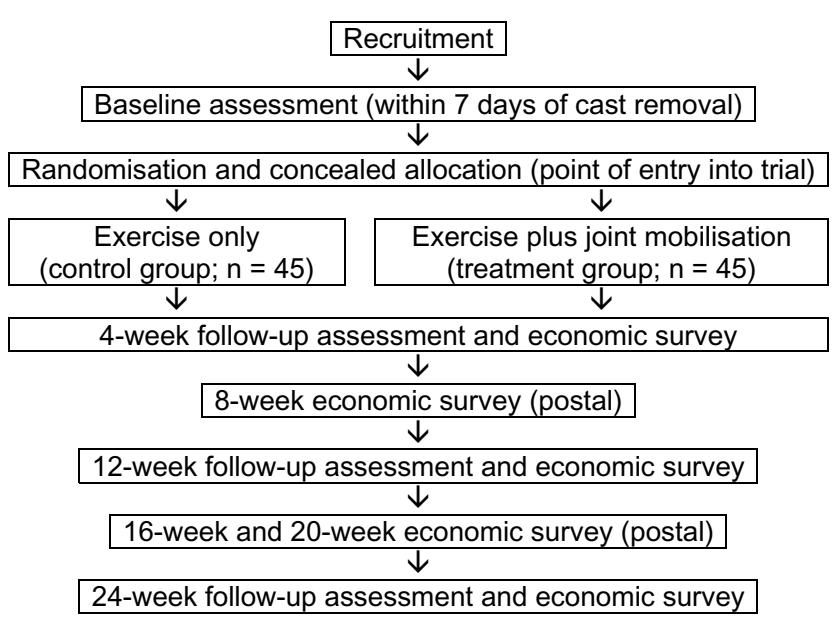

Figure I

Experimental protocol.

\section{Participants}

Participants will be recruited following cast removal from the plaster clinics and physiotherapy departments of three large teaching hospitals in Sydney, Australia (Royal North Shore Hospital, Royal Prince Alfred Hospital, and St Vincent's Public Hospital). The inclusion criteria will be:

- ankle fracture treated with cast immobilisation with or without surgical fixation

- cast removed in the preceding seven days

- approval from the orthopaedic specialist to weight-bear as tolerated or partial weight-bear

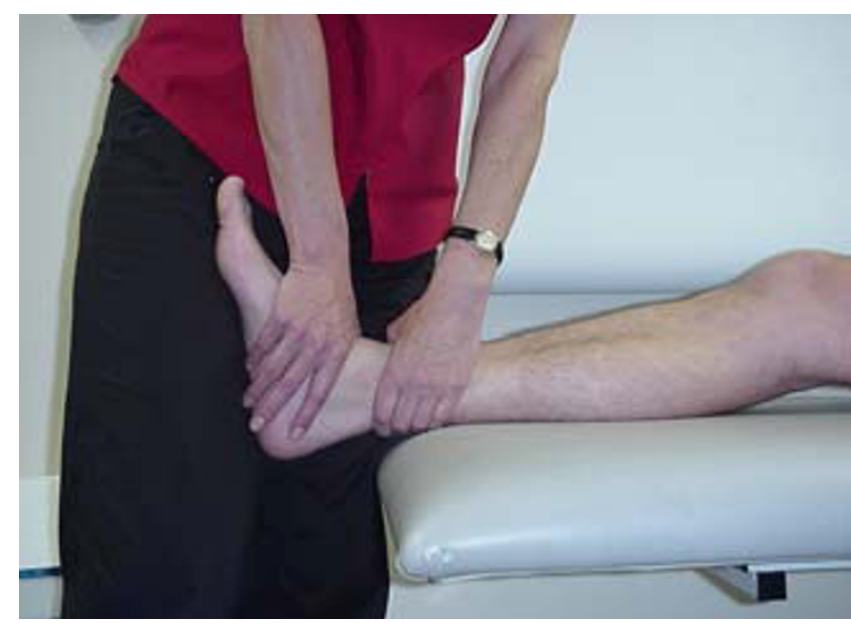

Figure 2

Passive joint mobilisation. The joint mobilisation technique used will be the anterior-posterior glide of the talus. The physiotherapist will apply large amplitude oscillatory movements into resistance (i.e., Grade III) on the talus.
- referral to outpatient physiotherapy for treatment

- at least 2 out of 10 pain in the ankle when up to $50 \%$ of body weight is borne through the affected leg

- completed skeletal growth assessed by the union of epiphyses on radiographs

- no concurrent and significant injuries or pathologies which may affect the recovery of the participant's lower limb function, and

- available for the six month follow-up period.

In addition to recruiting from multiple sites, other strategies will be used to maximise recruitment rates [40]. A log will be designed to aid the screening of potential participants at each site, and will be monitored by the investigators. Posters about the trial, inservices and regular communication with the participating physiotherapy departments will also assist to facilitate recruitment rates.

Ethical approval will be obtained from the ethics committee of each participating site (i.e., the University of Sydney Human Research Ethics Committee, Northern Sydney Health Human Research Ethics Committee, Sydney South West Area Health Service Ethics Review Committee (RPAH zone), and the St Vincent's Hospital Human Research Ethics Committee).

\section{Interventions}

Interventions will be delivered by registered physiotherapists, who will receive training to provide treatments in accordance with the experimental protocol.

Participants in the treatment group will receive passive joint mobilisation (see Figure 2). The joint mobilisation technique used will be the anterior-posterior glide of the talus [23]. The participant will be positioned in supine or long sitting, with the affected ankle moved to the end of the available range of pain-free dorsiflexion. The physiotherapist will manually apply large amplitude oscillatory movements into resistance (i.e., Grade III [23]) in an anterior-posterior direction on the talus for three sets of 60 seconds. This can be progressed by increasing the force and repetitions (up to five sets of 60 seconds) of the mobilisation, and the degree of ankle dorsiflexion. The choice of this technique is based on findings of a previous study, which showed that the anterior-posterior glide of the talus was more effective than usual care after ankle sprain [35].

Participants in both the treatment and control groups will be given an exercise programme that they will continue at home. Exercises will be chosen from a standardised set of exercises to ensure consistency. The exercises have been 
developed following consultation with physiotherapists at the recruiting hospitals and are based on the programme used in a previous study [41]. Three categories of exercise will be prescribed: ankle mobility and strengthening exercise, stepping exercise, and weight bearing and balancing exercise (see Figure 3). Participants will perform one exercise from each category at any one time. The treating physiotherapist will be responsible for the progression of the exercise programme, so the exercises will be tailored to the individual participant's needs and will always be challenging and difficult when repeated 10 times. Participants will be given exercise cards and exercise recording sheets to record the number of repetitions performed each day. The recommended frequency for most exercises will be three sets of 10 repetitions per exercise per day.

In this pragmatic trial, participants will be seen at a frequency typical to that which occurs in the clinical setting. Participants in the treatment group will receive two treatment sessions per week for four weeks for joint mobilisation and progression of the exercise programme. Participants allocated to the control group will receive two treatment sessions in the first week, then one session per week for three weeks. In the first session, participants in the control group will be taught the exercises, which will be monitored and progressed in subsequent sessions.

In addition, at the treatment sessions all participants will receive gait retraining, progression of walking aids, and advice on prognosis and return to activities. If required, participants will receive ice for pain relief, and compression and elevation for the management of swelling. No other physiotherapy treatments will be administered during the four-week intervention period.

After each initial treatment session, the treating physiotherapist will receive a phone call from an investigator unblinded to group allocation. Implementation of the experimental protocol for each participant will be discussed to ensure compliance.

At the end of the intervention period, passive joint mobilisation treatment will cease and participants will be allowed to be progressed to exercises other than those in the standardised set. Ankle taping can be performed to assist return to sports.

The timing of discharge will not be dictated in the protocol. Participants will be discharged by their physiotherapists when they return to their previous level of function, reach a plateau in their progress, or choose to discontinue treatment.

\section{Outcome assessment}

Participants will be assessed at baseline and the four, 12, and 24 week follow-up sessions by an assessor blinded to group allocation. The physiotherapists, participants and investigators will be asked to avoid giving information which may reveal group allocation to the blinded assessor. Assessor blinding will be checked after each follow-up assessment. The assessor will be asked if he/she was unblinded and will guess the allocation of each participant.

Demographic information will be collected and an X-ray assessment will be carried out by the blinded assessor at the baseline assessment. The X-ray assessment will determine the angle at which the fractured ankle was immobilised and the number of malleoli involved, in order to investigate the relationships between the angle of immobilisation and fracture severity with outcome.

The primary outcomes will be activity limitation and quality of life. They will be measured by the Lower Extremity Functional Scale [42] and the Assessment of Quality of Life scale [43], respectively. On the Lower Extremity Functional Scale, participants will rate the difficulty they have in performing 20 activities on a five-point scale from zero (extreme difficulty or unable to perform activity) to four (no difficulty). The maximum score is 80 points and a higher score denotes less limitation in activity. When tested in a sample of participants with lower limb injuries that included ankle fracture, the scale showed high internal consistency (alpha $=0.96$ ) and testretest reliability (intraclass correlation coefficient $=0.86$ ), and correlated well with the physical component of the Short Form 36 [42]. The Lower Extremity Functional Scale is capable of discriminating between patients requiring walking aides and patients not requiring walking aides, and has moderate correlations with weight-bearing status and ankle movement [44].

The Assessment of Quality of Life is designed to measure health-related quality of life, and has 15 questions covering five dimensions: illness, independent living, social relationships, physical senses and psychological wellbeing [43]. It has high internal consistency (alpha $=0.81$ ) [43]. The Assessment of Quality of Life is sensitive to changes in health states and is highly correlated with other commonly-used quality of life scales [46]. Population norms have been calculated for the Australian population [46].

Twelve secondary outcome measures will be used (Table 1). All participants will be given a calendar on which to mark the first day they can walk pain-free for 10 metres at a comfortable cadence. This will be used to calculate the number of days to pain-free walking from cast removal. 


\section{Ankle mobility \& strengthening exercises}

\section{Weight bearing $\&$ balancing exercises}

\begin{tabular}{|c|c|c|c|}
\hline$\cdot \frac{\pi}{\sqrt[n]{\pi}}$ & Theraband exercises & $\begin{array}{l}\text { Stepping forward and backward } \\
\text { with affected foot (standing on } \\
\text { affected foot) }\end{array}$ & $\begin{array}{l}\text { Taking as much } \\
\text { weight on unaffected } \\
\text { foot as possible }\end{array}$ \\
\hline & Heel raises on both feet & $\begin{array}{l}\text { Step-ups: affected foot on step, } \\
\text { unaffected foot stepping from } \\
\text { ground to step and back }\end{array}$ & $\begin{array}{l}\text { Standing on affected } \\
\text { leg only }\end{array}$ \\
\hline & $\begin{array}{l}\text { Heel raises with unaffected } \\
\text { foot on a chair, affected foot } \\
\text { on the ground }\end{array}$ & $\begin{array}{l}\text { Lateral stepping: affected foot on } \\
\text { step with medial border of foot } \\
\text { parallel to edge of step. Unaffected }\end{array}$ & $\begin{array}{l}\text { Standing on affected } \\
\text { leg with eyes closed }\end{array}$ \\
\hline & $\begin{array}{l}\text { Heel raises standing on } \\
\text { affected foot }\end{array}$ & $\begin{array}{l}\text { foot stepping sideways from ground } \\
\text { to step and back }\end{array}$ & $\begin{array}{l}\text { Standing on affected } \\
\text { leg on foam }\end{array}$ \\
\hline 范 & $\begin{array}{l}\text { Heel raises standing on } \\
\text { affected foot on a step }\end{array}$ & $\begin{array}{l}\text { Step-downs: affected foot on step, } \\
\text { unaffected foot stepping forward off } \\
\text { step to ground and back }\end{array}$ & $\begin{array}{l}\text { Standing on affected } \\
\text { leg on foam with eyes } \\
\text { closed }\end{array}$ \\
\hline
\end{tabular}

Figure 3

The home exercise programme. Participants will perform one exercise from each category of exercise. The physiotherapist will be responsible for the prescription, instruction, monitoring, and progression of the exercises.

Walking speed and step length asymmetry will be measured as the participants walk unaided along a 14-metre walkway. The average of three attempts will be used for data analysis. Participants will be instructed to walk as fast and as well as possible. Their time to walk the central 10 metres will be recorded by a stop watch, and walking speed will be calculated by dividing the distance covered by the time elapsed. A six-metre long, one-metre wide mat marked with five-centimetre grids will be taped to the floor over the central six metres of the walkway. Participants will be videotaped as they walk across the mat. Step length asymmetry (i.e., the difference in step length between the affected and unaffected sides) will be calcu- lated by playing the video recording in slow motion, and digitising the heel position of each footfall on a customised computer programme [47]. This method has high inter-rater, intra-rater and concurrent reliability [47-50].

Stepping rate on stairs will be calculated by the participants climbing four steps three times as fast as possible without using handrails. This task is part of the Motor Assessment Scale [51]. Although its psychometric properties have not been examined in people with musculoskeletal complaints, stair climbing will be included as an outcome measure as it is reasonably challenging and is a commonly-performed activity. 
Table I: Secondary outcome measures

\begin{tabular}{ll}
\hline $\begin{array}{l}\text { Number of days to pain-free walking } \\
\text { Measures of activity limitation: }\end{array}$ & Unaided walking speed \\
& Step length asymmetry during unaided walking \\
& Stepping rate on stair climbing \\
Measures of impairments: & Ankle dorsiflexion range of motion \\
& Pain on standing with equal weight distribution \\
Measures of participation: & Pain on stair descent \\
Global perceived effect of treatment & Return to usual work \\
Satisfaction with physiotherapy treatment & Return to sport and leisure activities \\
Negative effects of physiotherapy treatment &
\end{tabular}

Ankle dorsiflexion range of motion will be measured using the weight-bearing lunge method [52]. Participants will stand with the affected foot on the ground and the great toe against the wall, and will bend the knee to touch the wall. If the knee cannot reach the wall, the minimum distance between the knee and the wall without the heel lifting will be recorded in millimetres as a negative value. If the knee can touch the wall, the foot will be gradually moved back and the maximum distance between the great toe and the wall without the heel lifting will be recorded in millimetres as a positive value. Measured this way, ankle dorsiflexion at the time of cast removal has been shown to be a significant predictor of disability, perceived change in symptoms and active ankle dorsiflexion range six weeks and six months after ankle fracture [14]. The weight bearing lunge method has high inter-rater and intra-rater reliability (intraclass correlation coefficient 0.99 and 0.97 to 0.98 respectively) in the healthy population [52].

Pain on standing with equal weight distribution and during stair descent will be measured using a 100-mm visual analogue scale, labelled "no pain" on one end and "worst pain ever" on the other. The pain visual analogue scale correlates positively with other self-reported measures of pain intensity $[53,54]$. It has high test-retest reliability (intraclass correlation coefficient $=0.71$ to 0.99 ) [53], and is highly responsive in detecting change [54]. Participants will also rate their return to usual work, and return to usual sport and leisure activities using a 100-mm visual analogue scale, marked "not participating at all" at one end and "return to full level" at the other.

Global perceived effect of treatment will be measured on an 11-point scale, where participants rate their change in symptoms from -5 (vastly worse) to +5 (completely recovered). A scale of this kind is the most frequently used external criterion to which score changes on other outcome measurements are compared [42,55-57]. However its use as an external criterion is under debate [58,59], and there is little information regarding its validity and reliability [59].

Satisfaction with physiotherapy treatment will be measured at the four-week follow-up assessment on a 100-mm visual analogue scale marked "completely unsatisfactory" at one end and "best possible" at the other. At all followup assessments, participants will be asked if the physiotherapy treatment has had any negative effects and, if so, the nature of the effects. They will also record whether they have had treatment other than physiotherapy for the fractured ankle.

The treating physiotherapist will complete a survey for every participant at each follow-up assessment until discharge. Information gathered on this survey will be: the date and reason of discharge (if applicable), number of physiotherapy sessions scheduled, number of physiotherapy sessions attended, exercises prescribed and repetitions completed, any other physiotherapy treatment administered, and, for the treatment group only, the dates, number of sets and duration of the joint mobilisation treatment. This information will be analysed to obtain the length of physiotherapy required, percentage of scheduled sessions attended, compliance of participants to the exercise programme, and compliance of physiotherapists to the experimental protocol.

Data will be collected on paper forms and manually entered into a computer programme. Several strategies will be implemented to ensure the accuracy of the data. Data collection forms will be checked for completion. Data entry will be performed twice and the two entries will be compared to check for errors. Range checks will be performed on outcome values at the completion of data entry.

\section{Economic analysis}

The economic analysis will be conducted from the perspective of the Australian healthcare system and the indi- 


\section{Type of Resource}

Physiotherapy costs

Equipment

Participants' out-of-pocket costs for

interventions or medication in relation to

the ankle fracture

Use of other healthcare resources in

relation to the ankle fracture (e.g. visits to

specialists and alternative health services)
Source of data

Experimental protocol

and observation in the

hospital settings

Experimental protocol

4-weekly surveys over 24

weeks

\section{4-weekly surveys over 24}

weeks and cost to the Australian

health system

\section{Method of valuation}

Salary rates and on-costs for

physiotherapists

Manufacturer's price

(depreciated over 3 years)

Actual costs to participants

and cost to the Australian

health system

Actual costs to participants

\section{Figure 4}

Economic evaluation

vidual patient, with data collected in a survey every four weeks from the end of the four-week intervention period to the 24-week follow-up assessment. Participants will record all out-of-pocket expenses incurred due to the fractured ankle, including the frequency and costs associated with physiotherapy, other health and hospital services, and the costs of medication. Figure 4 indicates the type of resources which will be captured, the sources of data, and proposed methods of valuation. At the four-week followup assessment, participants will also be asked to provide socio-demographic information such as their usual main activity, normal annual household income, the type of private health insurance they have (if any), and whether they are renting, boarding or owners of their homes.

\section{Statistical analysis}

Data analysis will be undertaken at the completion of data acquisition and will be by intention to treat (that is, participants will be analysed according to group allocation regardless of compliance) [60]. Missing data will be replaced by the last known value carried forward. The statistician will be given coded data to ensure blinding.

Separate analyses will be performed on the four, 12 and 24 week data, with the four-week (i.e., the end of the intervention period) follow-up being the primary time point for analysis. To test the effects of treatment, betweengroup differences will be examined with analysis of covariance using a regression approach. The emphasis will be 
on estimation, but hypothesis tests will also be conducted (alpha $=0.05)$. Pre-test scores will be entered into the model as the only covariate.

In a secondary analysis designed to test the influence of fracture severity on treatment effects, additional terms (fracture severity, group by fracture severity interaction) will be entered into the regression model. Fracture severity will be categorised into two levels based on the number of malleoli involved: less severe (unimalleolar fractures) and more severe (bimalleolar or trimalleolar fractures). The effect of fracture severity on treatment will be determined by examining the interactions between group of allocation and fracture severity.

Ninety participants (45 per group) will participate in the trial. This sample size provides a $90 \%$ probability of detecting a difference between group means of 10 points on the Lower Extremity Functional Scale, assuming a standard deviation of 12 based on a recently published study [41]. These calculations assume a correlation of 0.6 between pre- and post-test measures and an alpha of 0.05 , and allowed for $10 \%$ dropouts and $20 \%$ non-compliance.

The economic evaluation will consist of cost-effectiveness and cost-utility analyses. The cost-effectiveness analysis will use the Lower Extremity Functional Scale as a measure of effectiveness. The cost-utility analysis will use the Assessment of Quality of Life as a measure of utility [43]. The analyses will examine differences between treatment and control participants in terms of costs incurred and reductions in perceived disability (cost-effectiveness analysis) or utility gained (cost-utility analysis).

The incremental cost-effectiveness (utility) ratio (ICER) will be calculated as: ICER $=\left(\mathrm{C}_{\mathrm{E}}-\mathrm{C}_{\mathrm{C}}\right) /\left(\mathrm{U}_{\mathrm{E}}-\mathrm{U}_{\mathrm{C}}\right)$, where $\mathrm{C}$ is average cost, $\mathrm{U}$ is the average effectiveness or utility score, and subscripts $\mathrm{E}$ and $\mathrm{C}$ denote the treatment and control arms. Joint mobilisation can be said to be costeffective relative to exercise alone if it produces less perceived disability and/or greater utility at a lower cost, or the cost per disability avoided or per quality of life gained (i.e., the ICER) is less than some threshold value (e.g., $\$ 50,000$ in Australian dollars). Sensitivity analysis will be undertaken to explore the robustness and validity of the results. That is, both costs and outcomes will be varied in line with results from similar studies reported in the literature and the upper and lower bounds of the results from this trial.

\section{Funding}

The trial is funded by the Motor Accidents Authority of New South Wales, Australia (grant reference number 04/ 241).

\section{Discussion}

The primary aim of this randomised controlled trial is to determine the effectiveness and cost-effectiveness of passive joint mobilisation, in addition to an exercise programme, in adults after cast immobilisation for ankle fracture. The trial incorporates design features known to minimise bias [62]. Participants will be assigned to treatment or control groups using a concealed random procedure. Assessments will be blinded. Data analysis will also be blinded and by intention to treat.

Blinding confers several potential benefits to the rigor of randomised controlled trials, particularly minimising bias $[61,62]$. Failure to implement blinding could lead to inflated estimates of effects $[62,63]$. The nature of the treatments involved in this trial means that blinding of the physiotherapists and participants is not possible. Nevertheless, satisfaction with physiotherapy will be evaluated as a means to assess participants' perception of the treatment received.

A pragmatic trial design has been chosen as it can directly inform clinical practice [64-67]. The inclusion criteria of the trial reflect the variety in patient presentations that would be encountered by physiotherapists in the clinical setting. The experimental protocol also simulates clinical practice. Participants will be treated with the same frequency and for the same duration as would be normal practice, and one form of treatment (joint mobilisation plus exercise) will be compared to another form of treatment (exercise only), instead of placebo. The outcome measures chosen are clinically oriented and participants will be analysed according to group allocation regardless of compliance. Using a pragmatic design broadens the generalisability of the results. An added advantage to this trial is that the pragmatic design allows a realistic economic evaluation of the two treatment alternatives [65].

It is anticipated that data acquisition will finish by early 2007. Results from this trial will contribute to evidencebased guidelines for rehabilitation after ankle fracture.

\section{Competing interests}

The author(s) declare that they have no competing interests.

\section{Authors' contributions}

$\mathrm{AM}, \mathrm{KR}, \mathrm{MH}$ and $\mathrm{RH}$ conceived and designed the trial protocol, and procured funding. MH designed the economic analysis. RH designed the statistical analysis. CL refined and developed the trial protocol. CL implemented the trial protocol, managed the trial, performed blinded outcome assessment and data entry, and drafted the manuscript. $\mathrm{AM}, \mathrm{KR}, \mathrm{MH}$ and $\mathrm{RH}$ contributed to the 
manuscript. All authors read and approved the final manuscript.

\section{Acknowledgements}

This trial is funded by the Motor Accidents Authority of New South Wales, Australia. The authors would like to acknowledge the associate investigators of the trial: Trish Evans and Deborah Taylor at Royal North Shore Hospital, Sandeep Gupta and Louise Shaw at Royal Prince Alfred Hospital, and Steve Bradley and Bruce Donald at St Vincent's Public Hospital. The authors are grateful to the trial participants and the physiotherapy departments at Royal North Shore Hospital, Royal Prince Alfred Hospital, and St Vincent's Public Hospital, particularly: Craig Gregory, Carmel Bertuccio, Julie Penn, Nick Green and Michelle Skeed.

\section{References}

I. Whittle PA, Wood GW: Fractures of lower extremity. In Campbell's operative orthopaedics Volume 3. I0th edition. Edited by: Canale ST. Philadelphia, Mosby; 2003:2725-2872.

2. Michelson JD: Current concepts review. Fractures about the ankle. The Journal of bone and joint surgery American volume 1995, 77 A: $142-152$.

3. Donatto KC: Ankle fractures and syndesmosis injuries. Orthop Clin North Am 2001, 32:79-90.

4. Lesic A, Bumbasirevic M: Ankle fractures. Curr Orthop 2004, 18:232-244.

5. Bengner U, Johnell O, Redlund-Johnell I: Epidemiology of ankle fracture 1950 and 1980. Increasing incidence in elderly women. Acta Orthop Scand 57(I):35-7 1986.

6. Court-Brown CM, McBirnie J, Wilson G: Adult ankle fractures an increasing problem? Acta Orthop Scand 69(I):43-7 1998, 69:43-47.

7. Daly PJ, Fitzgerald RHJ, Melton LJ, Ilstrup DM: Epidemiology of ankle fractures in Rochester, Minnesota. Acta Orthopaedica Scandinavica 1987, 58:539-544.

8. Jensen SL, Andresen BK, Mencke S, Nielsen PT: Epidemiology of ankle fractures: a prospective population-based study of $2 / 2$ cases in Aalborg, Denmark. Acta Orthop Scand 1998, 69:48-50.

9. Lash N, Horne G, Fielden J, Devane P: Ankle fractures: functional and lifestyle outcomes at 2 years. ANZ Journal of Surgery 2002, 72:724-730.

10. Ward EG, Bodiwala GG, Thomas PD: The importance of lower limb injuries in car crashes when cost and disability are considered. Accident; Analysis and Prevention 1992, 24:613-620.

II. Solomon L, Warwick D, Nayagam S: Principles of fractures. In Apley's system of orthopaedics and fractures 8th edition. London, Arnold; 200I:539-582.

12. Solomon L, Warwick D, Nayagam S: Injuries of the ankle and foot. In Apley's system of orthopaedics and fractures 8th edition. London, Arnold; $2001: 733-759$.

13. Bewes PC: The management of ankle fractures. Trop Doct 1995, 25:58-62.

14. Hancock MJ, Herbert RD, Stewart M: Prediction of outcome after ankle fracture. J Orthop Sports Phys Ther 2005, 35:786-792.

15. Broos PL, Bisschop AP: Operative treatment of ankle fractures in adults: correlation between types of fracture and final results. Injury 1991, 22:403-406.

16. Chesworth BM, Vandervoort AA: Comparison of passive stiffness variables and range of motion in uninvolved and involved ankle joints of patients following ankle fractures. Physical Therapy 1995, 75:253-26I.

17. Shaffer MA, Okereke E, Esterhai JLJ, Elliott MA, Walker GA, Yim SH, Vandenborne K: Effects of immobilization on plantar-flexion torque, fatigue resistance, and functional ability following an ankle fracture. Physical Therapy 2000, 80:769-780.

18. Stevens JE, Walter GA, Okereke E, Scarborough MT, Esterhai IL, George SZ, Kelley MJ, Tillman SM, Gibbs JD, Elliott MA, Frimel TN, Gibbs CP, Vandenborne K: Muscle adaptations with immobilization and rehabilitation after ankle fracture. Med Sci Sports Exerc 36(10):1695-70I 2004.

19. Vandenborne K, Elliott MA, Walter GA, Abdus S, Okereke E, Shaffer $M$, Tahernia $D$, Esterhai JL: Longitudinal study of skeletal muscle adaptations during immobilization and rehabilitation. Muscle \& Nerve 1998, 21:1006-1012.

20. Belcher GL, Radomisli TE, Abate JA, Stabile LA, Trafton PG: Functional outcome analysis of operatively treated malleolar fractures. J Orthop Trauma II (2): 106-9 1997, I I:106-109.

21. Nilsson G, Nyberg P, Ekdahl C, Eneroth M: Performance after surgical treatment of patients with ankle fractures - I4-month follow-up. Physiother Res Int 2003, 8:69-82.

22. Ponzer S, Nasell H, Bergman B, Tornkvist H: Functional outcome and quality of life in patients with Type $B$ ankle fractures: a two-year follow-up study. J Orthop Trauma 13(5):363-8 1999.

23. Maitland GD: Peripheral manipulation. 3rd edition. London, Boston, Butterworth-Heinemann; I99I.

24. Lederman E: Fundamentals of manual therapy. New York, Churchill Livingstone; 1997.

25. Refshauge KM: Selection of treatment for musculoskeletal conditions. In Musculoskeletal physiotherapy: clinical science and evidence-based practice 2 nd edition. Edited by: Refshauge KM and Gass EM. Oxford, Butterworth-Heinemann; 2004:245-275.

26. Sluka KA, Wright $A$ : Knee joint mobilization reduces secondary mechanical hyperalgesia induced by capsaicin injection into the ankle joint. European Journal of Pain 200I, 5:8I-87.

27. Paungmali A, O'Leary S, Souvlis T, Vicenzino B: Naloxone fails to antagonize initial hypoalgesic effect of a manual therapy treatment for lateral epicondylalgia. Journal of Manipulative \& Physiological Therapeutics 2004, 27: $180-185$

28. Paungmali A, O'Leary S, Souvlis T, Vicenzino B: Hypoalgesic and sympathoexcitatory effects of mobilization with movement for lateral epicondylalgia. Physical Therapy 2003, 83:374-383.

29. Simon $R$, Vicenzino $B$, Wright $A$ : The influence of an anteroposterior accessory glide of the glenohumeral joint on measures of peripheral sympathetic nervous system function in the upper limb. Manual Therapy 1997, 2: 18-23.

30. Randall T, Portney L, Harris BA: Effects of joint mobilization on joint stiffness and active motion of the metacarpal-phalangeal joint. J Orthop Sports Phys Ther 1992, 16:30-36.

31. Coyle JA, Robertson V]: Comparison of two passive mobilizing techniques following Colles' fracture: a multi-element design. Manual Therapy 1998, 3:34-4I.

32. Taylor NF, Bennell KL: The effectiveness of passive joint mobilisation on the return of active wrist extension following Colles' fracture: a clinical trial. New Zealand Journal of Physiotherapy 1994, 22:24-28.

33. Kay S, Haensel N, Stiller K: The effect of passive mobilisation following fractures involving the distal radius: a randomised study. Australian Journal of Physiotherapy 2000, 46:93-101.

34. van der Wees PJ, Lenssen AF, Hendricks EJM, Stomp DJ, Dekker J, de Bie RA: Effectiveness of exercise therapy and manual mobilisation in acute ankle sprain and functional instability: A systematic review. Australian Journal of Physiotherapy 2006, 52:27-37.

35. Green T, Refshauge K, Crosbie J, Adams R: A randomized controlled trial of a passive accessory joint mobilization on acute ankle inversion sprains. Physical Therapy 200I, 81 : 984-994.

36. Wilson FM: Manual therapy versus traditional exercises in mobilisation of the ankle post-ankle fracture: a pilot study. New Zealand Journal of Physiotherapy 1991, 19:1 I-16.

37. James LA, Sookhan N, Subar D: Timing of operative intervention in the management of acutely fractured ankles and the cost implications. Injury 200I, 32:469-472.

38. Bhandari M, Sprague S, Ayeni OR, Hanson BP, Moro JK: A prospective cost analysis following operative treatment of unstable ankle fractures: $\mathbf{3 0}$ patients followed for I year. Acta Orthopaedica Scandinavica 2004, 75: $100-105$.

39. van den Hout WB, Vermeulen HM, Rozing PM, Vlieland TP: Impact of adhesive capsulitis and economic evaluation of high-grade and low-grade mobilisation techniques. Australian Journal of Physiotherapy 2005, 51: I4I-I49.

40. Lovato LC, Hill K, Hertert S, Hunninghake DB, Probstfield JL: Recruitment for controlled clinical trials: literature summary and annotated bibliography. Controlled Clinical Trials 1997, 18:328-352.

41. Moseley AM, Herbert RD, Nightingale EJ, Taylor DA, Evans TM, Robertson GJ, Gupta SK, Penn J: Passive stretching does not enhance outcomes in patients with plantarflexion contracture after cast immobilization for ankle fracture: $A$ rand- 
omized controlled trial. Arch Phys Med Rehabil 2005, 86: $1118-1126$

42. Binkley JM, Stratford PW, Lott SA, Riddle DL: The Lower Extremity Functional Scale (LEFS): scale development, measurement properties, and clinical application. North American Orthopaedic Rehabilitation Research Network. Physical Therapy 1999, 79:37I-383.

43. Hawthorne G, Richardson J, Osborne R: The Assessment of Quality of Life (AQoL) instrument: a psychometric measure of health-related quality of life. Quality of Life Research 1999, 8:209-224.

44. Alcock GK, Stratford PW: Validation of the Lower Extremity Functional Scale on athletic subjects with ankle sprains. Physiother Can 2002, 54:233-240.

45. Hawthorne G, Richardson J, Day NA: A comparison of the Assessment of Quality of Life (AQoL) with four other generic utility instruments. Annals of Medicine 200I, 33:358-370.

46. Hawthorne G, Osborne R: Population norms and meaningful differences for the Assessment of Quality of Life (AQoL) measure. Aust N Z J Public Health 2005: I36-142.

47. Wall JC, Devlin J, Khirchof R, Lackey B: Measurement of step widths and step lengths: a comparison of measurements made directly from a grid with those made from a video recording. J Orthop Sports Phys Ther 2000, 30:410-4I7.

48. Gaudet G, Goodman R, Landry M, Russell G, Wall JC: Measurement of step length and step width: a comparison of videotape and direct measurements. Physiother Can 1990, 42: I 2-15.

49. van Loo MA, Moseley AM, Bosman JM, de Bie RA, Hassett L: Testre-test reliability of walking speed, step length and step width measurement after traumatic brain injury: a pilot study. Brain Injury 2004, I 8: 104I-I048.

50. van Loo MA, Moseley AM, Bosman JM, de Bie RA, Hassett L: Interrater reliability and concurrent validity of step length and step width measurement after traumatic brain injury. Disability and Rehabilitation 2003, 25: I 195- 1200.

5I. Carr JH, Shepherd RB, Nordholm L, Lynne D: Investigation of a new motor assessment scale for stroke patients. Physical Therapy 1985, 65: 175-180.

52. Bennell K, Talbot RC, Wajswelner H, Techovanich W, Kelly DH, Hall AJ: Intra-rater and inter-rater reliability of a weight-bearing lunge measure of ankle dorsiflexion. Australian Journal of Physiotherapy 1998, 44:175-180.

53. Kahl C, Cleland JA: Visual analogue scale, numeric pain rating scale and the McGill pain Questionnaire: an overview of psychometric properties. Phys Ther Rev 2005, 10:123-128.

54. von Korff M, Jensen MP, Karoly P: Assessing global pain severity by self-report in clinical and health services research. Spine 2000, 25:3|40-3|5|.

55. Beurskens AJ, de Vet HC, Koke AJ: Responsiveness of functional status in low back pain: a comparison of different instruments. Pain 1996, 65:71-76.

56. Hagg O, Fritzell P, Nordwall A, Swedish Lumbar Spine Study Group: The clinical importance of changes in outcome scores after treatment for chronic low back pain. European Spine Journal 2003, I 2: $12-20$.

57. Pengel LH, Refshauge KM, Maher CG: Responsiveness of pain, disability, and physical impairment outcomes in patients with low back pain. Spine 2004, 29:879-883.

58. Fritz JM, Irrgang J]: A comparison of a modified Oswestry Low Back Pain Disability Questionnaire and the Quebec Back Pain Disability Scale. Physical Therapy 200I, 81:776-788.

59. Norman GR, Stratford P, Regehr G: Methodological problems in the retrospective computation of responsiveness to change: the lesson of Cronbach. J Clin Epidemiol 50(8):869-79 1997.

60. Hollis S, Campbell $\mathrm{F}$ : What is meant by intention to treat analysis? Survey of published randomised controlled trials. $B M J$ 1999, 31 19:670-674.

61. Schulz KF, Chalmers I, Hayes RJ, Altman DG: Empirical evidence of bias: dimensions of methodological quality associated with estimates of treatment effects in controlled trials. JAMA : the journal of the American Medical Association 1995, 273:408-4I2.

62. Schulz KF, Grimes DA: Blinding in randomised trials: hiding who got what. Lancet 2002, 359:696-700.

63. Boutron I, Estellat C, Ravaud P: A review of blinding in randomized controlled trials found results inconsistent and questionable. Journal of Clinical Epidemiology 2005, 58:1220-1226.
64. Helms PJ: 'Real world' pragmatic clinical trials: what are they and what do they tell us? Pediatric allergy and immunology 2002, 13:4-9.

65. Macpherson H: Pragmatic clinical trials. Complementary Therapies in Medicine 2004, I 2: 136- I 70

66. Roland M, Torgerson DJ: Understanding controlled trials: What are pragmatic trials? $B M \mid$ | 998,3 | 6:285

67. Tunis SR, Stryer DB, Clancy CM: Practical clinical trials. Increasing the value of clinical research for decision making in clinical and health policy. JAMA : the journal of the American Medical Association 2003, 290: 1624-1632.

\section{Pre-publication history}

The pre-publication history for this paper can be accessed here:

http://www.biomedcentral.com/1471-2474/7/46/prepub
Publish with Bio Med Central and every scientist can read your work free of charge

"BioMed Central will be the most significant development for disseminating the results of biomedical research in our lifetime. "

Sir Paul Nurse, Cancer Research UK

Your research papers will be:

- available free of charge to the entire biomedical community

- peer reviewed and published immediately upon acceptance

- cited in PubMed and archived on PubMed Central

- yours - you keep the copyright
BioMedcentral 